\title{
Effect of chlorination and ozone pre-oxidation on the photobacteria acute toxicity for dissolved organic matter from sewage treatment plants
}

\author{
ZHANG Hua, QU JiuHui \& LIU HuiJuan* \\ State key Laboratory of Environmental Aquatic Chemistry; Research Center for Eco-Environmental Sciences, \\ Chinese Academy of Sciences, Beijing 100085, China
}

Received January 7, 2010; accepted May 27, 2010

\begin{abstract}
The effect of chlorination and ozone pre-oxidation on the photobacteria acute toxicity for dissolved organic matter (DOM) from sewage treatment plants was investigated in this study. The results show that ozone pre-oxidation enhanced the photobacteria acute toxicity of the water samples. DOM before and after ozone pre-oxidation was fractionated by resins into six kinds of hydrophobic and hydrophilic organics. The six fractions were chlorinated individually and the photobacteria acute toxicity before and after chlorination was tested. It was found that the percentage of hydrophilic organics in DOM significantly increased after ozone pre-oxidation and hydrophilic organics exhibited remarkably higher acute toxicity than hydrophobic organics. In view of potentiometric titration and fourier transform infrared (FTIR) analysis, the hydrophilic organics showed a rather higher content of ph-OH structures than hydrophobic organics.
\end{abstract}

photobacteria acute toxicity, DOM, hydrophilic organics, hydrophobic organics

\section{Introduction}

Wastewater reuse has been regarded as an important method to alleviate the serious shortage of water resources, and it is significant to ensure the biological, ecological and environmental safety during wastewater reclamation [1]. Photobacterium bioassay method can quantify the decrease in light emission from the photobacteria as a result of exposure to pollutants in a short time. The toxicity test which is rapid and easy to perform has been widely used to assess the toxicity of drinking water and wastewater.

Dissolved organic matter (DOM) in the complex reclaimed water can react with chlorine to produce toxic disinfection by-products (DBPs) during the chlorine disinfection process [2-6]. In order to decrease the formation of

*Corresponding author (email: hjliu@ @rcees.ac.cn)
DBPs, advanced treatment methods are used in wastewater reuse to remove the precursors for DBPs. Ozonation as a pre-oxidation treatment has been widely investigated; however, studies about the effect of ozone pre-oxidation on the acute toxicity of DOM in the reclaimed water are still limited.

In this study, the effect of ozone pre-oxidation and chlorination on the photobacteria toxicity of DOM was investigated. In addition, DOM was fractionated into six fractions using resins and the relationship between the acute toxicity and DOM fractions was examined.

\section{Materials and methods}

\subsection{Collection of water samples}

Water samples were collected from Gaobeidian sewage treatment plant that incorporated activated sludge treatment process and advanced treatment, such as enhanced coagula- 
tion and sand-filtration. The effluent was mostly used as reclaimed water without further treatment. Water samples for this study were collected at the sand filtration outlet then filtered immediately through a pre-rinsed $0.45 \mu \mathrm{m}$ cellulose filter and stored in the dark at $4{ }^{\circ} \mathrm{C}$ until analysis.

\subsection{Fractionation of DOM by resin adsorption}

Three resin adsorbents, i.e., Amberlite XAD-8 resin (20-60 mesh), Dowex Marathon MSC resin (20-50 mesh) and Duolite A-7 (free base), were used to isolate DOM into six fractions: hydrophobic acids (HoA), hydrophobic bases (HoB), hydrophobic neutrals (HoN), hydrophilic acids (HiA), hydrophilic bases $(\mathrm{HiB})$ and hydrophilic neutrals $(\mathrm{HiN})$. The fractionation procedure of DOM was shown in Figure 1.

\subsection{Analytical methods}

Dissolved organic carbon (DOC) was measured with a TOC analyzer (Multi N/C 3000) in accordance with Standard Method 5310D.

DOM powder obtained through freeze-drying the fractions was analyzed for the structural and chemical characteristics of DOM. KBr was mixed with the DOM powder at the ratio of 100 to 1 and the FTIR spectra of the mixture were obtained by scanning it with a FTIR spectrometer (Thermo Nicolet NEXUS 670, USA).

The content of $-\mathrm{COOH}$ and ph-OH in DOM was tested using a potentiometric titration meter (Metrohm Co., Switzerland).

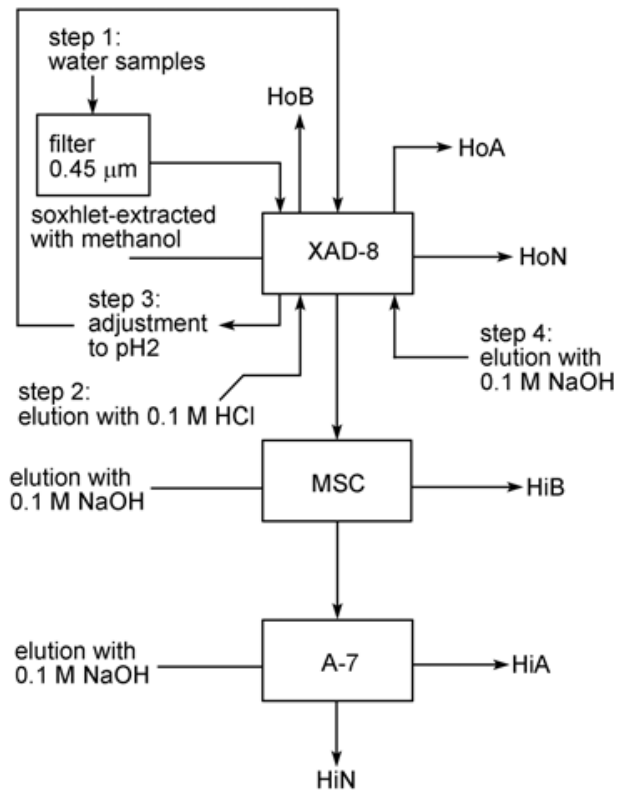

Figure 1 Schematic diagram of the procedure for DOM fractionation. HoB, Hydrophobic bases; HoA, hydrophobic acids; HoN, hydrophobic neutrals; HiB, hydrophilic bases; HiA, hydrophilic acids; HiN, hydrophilic neutrals.

\subsection{Ozone pre-oxidation}

Ozonation tests were performed using a semi-continuous system which included an oxygen generator (Boyikang Co., Beijing), an ozone generator (Mitsubishi, Japan) and a 3-liter glass reactor fitted with a magnetic impeller. With a flow rate of about $150 \mathrm{~mL} / \mathrm{min}$, ozone was added into water samples with a dosage of $1.8 \mathrm{mg} / \mathrm{min}$. Excess ozone was passed into a gas absorption bottle containing $2 \% \mathrm{KI}$ solution.

\subsection{Chlorination}

Water samples which were conditioned to $\mathrm{pH} 7$ by $2 \mathrm{~mol} / \mathrm{L}$ $\mathrm{HCl}$ and $2 \mathrm{~mol} / \mathrm{L} \mathrm{NaOH}$ were collected in $50 \mathrm{~mL}$ tubes individually and $1 \mathrm{~mL}$ of $50 \mathrm{mM}$ sodium phosphate buffer solution ( $\mathrm{pH} 7$ ) was added. Then, free chlorine $(\mathrm{HOCl})$ stock solution (about $5 \mathrm{~g} / \mathrm{L}$ ) was added. Following incubation in the dark at $20{ }^{\circ} \mathrm{C}$ for three days, the chlorination was quenched with sodium sulfite $\left(\mathrm{Na}_{2} \mathrm{SO}_{3}\right)$ and DBPs were analyzed.

Hexane and methyl tert-butyl ether used to extract DBPs were obtained from Fisher and J.T.Baker, respectively. Four THMs standards, i.e., chloroform $\left(\mathrm{CHCl}_{3}\right)$, bromodichloromethane $\left(\mathrm{CHCl}_{2} \mathrm{Br}\right)$, dibromochloromethane $\left(\mathrm{CHClBr}_{2}\right)$ and bromoform $\left(\mathrm{CHBr}_{3}\right)$, were determined according to EPA method 551.1 [7], using an Agilent 6890N Gas Chromatograph (USA) that was equipped with a fused silica capillary column (HP-5, $30 \mathrm{~m}, 320 \mu \mathrm{m} \times 0.25 \mu \mathrm{m})$ and a linearized electron capture detector (ECD). Five HAAs standards, i.e., monochloroacetic acid (MCAA), dichloroacetic acid (DCAA), trichloroacetic acid (TCAA), monobromoacetic acid (MBAA), and dibromoacetic acid (DBAA), were analyzed according to EPA Method 552.3 [8].

\subsection{Photobacteria acute toxicity test [9]}

The bacteria were provided as freeze-dried powder by the Institute of Soil Science, Chinese Academy of Sciences, China. The luminescence intensity was determined by a test instrument (Model toxicity analyzer DXY-2, China). All experiments were repeated more than 3 times.

In order to explore the effect of chlorination and ozone pre-oxidation on photobacteria acute toxicity, water samples without any treatment were used as blanks:

Relative luminescence intensity $(\%)=$ luminescence intensity after treatment/luminescence intensity before treatment $\times 100 \%$.

Higher relative luminescence intensity means that the photobacteria acute toxicity of the sample is lower.

\section{Results and discussion}

\subsection{Effect of chlorination and ozone pre-oxidation on the photobacteria acute toxicity of DOM}

DOM in the reclaimed water sample was chlorinated before 
and afeer ozonation, respetively, then the photobacteria acute toxicity was tested. Figure 2 showed the relative luminescence intensity of DOM with different treatment methods. It was observed that ozone pre-oxidation significantly enhanced the acute toxicity of DOM.

As shown in Figure 3, toxic DBPs formed after chlorination, whereas the increase in photobacteria acute toxicity caused by chlorination was not as significant as that by ozone pre-oxidation. In addition, the acute toxicity of DOM after ozone pre-oxidation was reduced by the subsequent chlorination.

Therefore, it can be concluded that both DOM and DBPs were toxic to photobacteria, while the variation in DOM structures caused by ozonation resulted in remarkably higher acute toxicity than chlorination.

\subsection{Variation of DOM structures caused by ozonation}

In order to explore the effect of DOM structures on photobacteria acute toxicity, ozone was continuously introduced into DOM for $30 \mathrm{~min}$ and resins were used to fractionate DOM before and after ozone pre-oxidation. The relationship of ozone pre-oxidation with DOM fractions was presented in

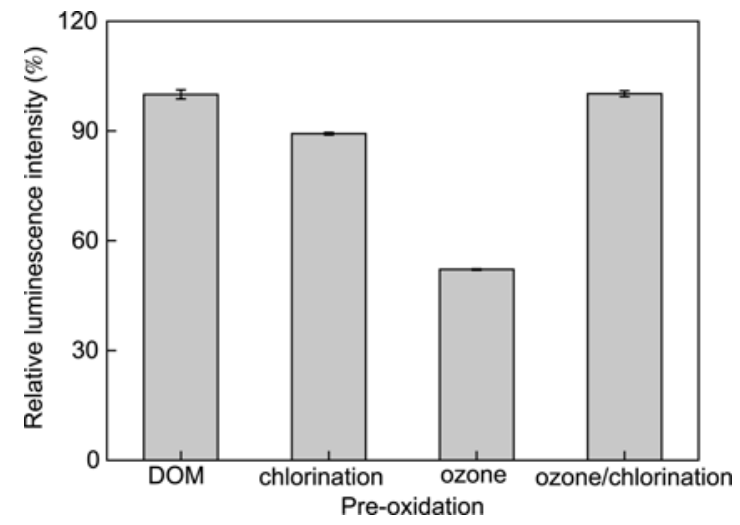

Figure 2 Relative luminescence intensity when DOM was chlorinated before and afeer ozonation (the luminescence intensity for DOM without treatment was set as $100 \%$ ). Higher relative luminescence intensity means that the photobacteria acute toxicity of the sample is lower.

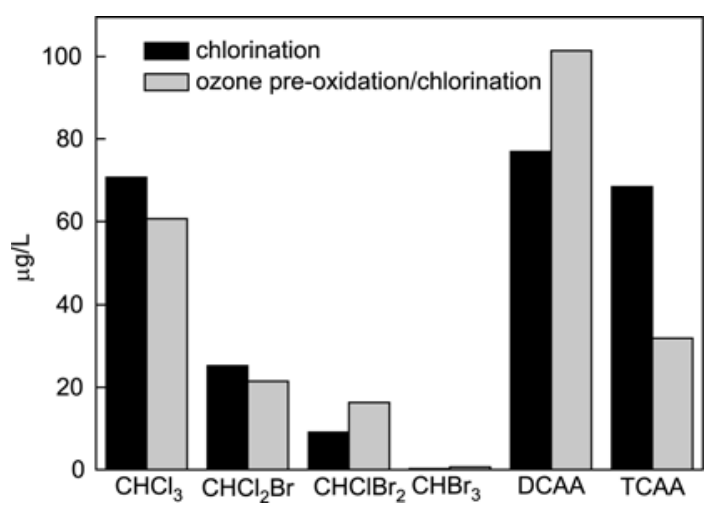

Figure 3 Formation potential of DBPs of DOM before and after ozone pre-oxidation.
Figure 4.

It was found that after ozone pre-oxidation, the DOC percentage of hydrophobic organics in DOM dropped from $51.9 \%$ to $34.5 \%$, whereas a substantial increase from $48.1 \%$ to $65.5 \%$ in the percentage of hydrophilic organics was observed. Moreover, the percentage of HiA after the ozonation was about 2.3 times of that before ozone pre-oxidation and the percentage of $\mathrm{HiN}$ was also increased by $20 \%$.

In the study, dominant fractions of DOM, i.e., organic acids (HoA, HiA) and organic neutrals ( $\mathrm{HoN}, \mathrm{HiN})$, were mainly examined. In order to compare the characteristics of DOM fractions, all samples were conditioned to similar DOC concentrations about $3 \mathrm{mg} / \mathrm{L}$ and $\mathrm{pH} 7-8$. The photobacteria acute toxicity for the four DOM fractions was investigated before and after chlorination (Figure 5). Table 1 showed the formation potential of DBPs of the four fractions.

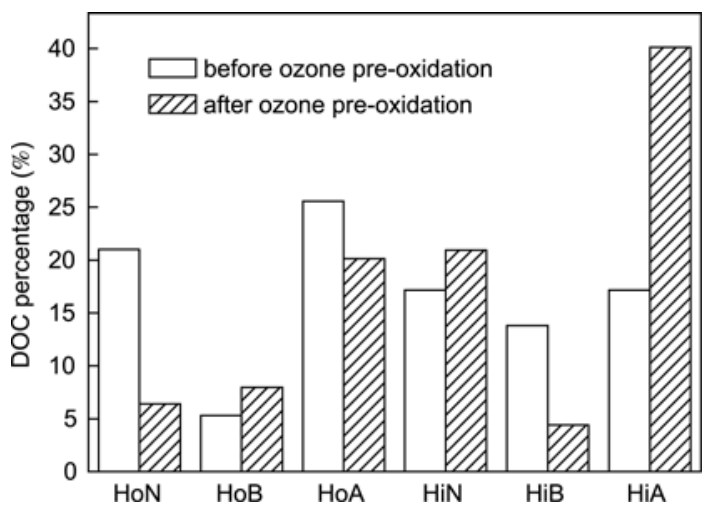

Figure 4 DOC percentage of DOM fractions before and after ozone pre-oxidation.

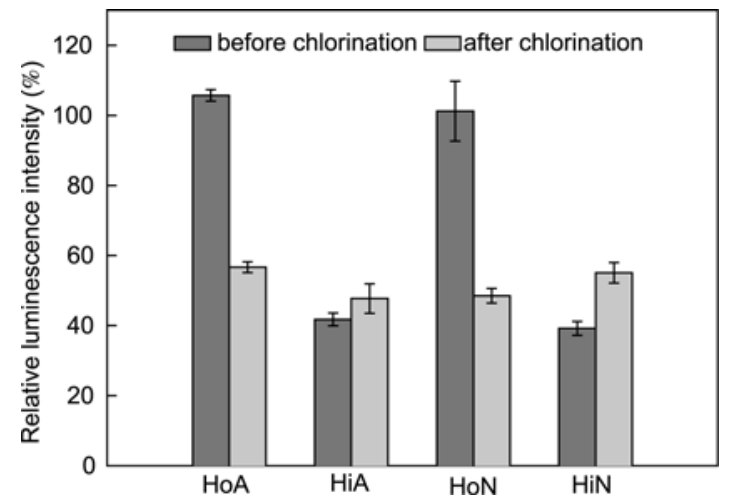

Figure 5 Photobacteria acute toxicity of DOM fractions before and after chlorination.

Table 1 Formation potential of DBPs of DOM fractions

\begin{tabular}{cccc}
\hline & $\mathrm{UV}_{254}$ & THM $(\mu \mathrm{g} / \mathrm{mgC})$ & HAA $(\mu \mathrm{g} / \mathrm{mgC})$ \\
\hline HoA & 0.047 & 53.94 & 28.16 \\
HiA & 0.042 & 38.87 & 23.06 \\
HoN & 0.055 & 34.67 & 10.50 \\
HiN & 0.022 & 27.85 & 21.4 \\
\hline
\end{tabular}


Hydrophobic organics exhibited relatively low acute toxicity prior to chlorination, whereas a significant increase in acute toxicity was observed after chlorination. However, the relative luminescence intensity for hydrophilic organics was about $40 \%$ before chlorination, which was lower than one half of that for hydrophobic organics. In addition, the relatively strong acute toxicity for hydrophilic organics was hardly altered by chlorination. Thus, it can be concluded that hydrophobic organics formed certain DBPs during chlorination which was rather toxic to photobacteria; however, hydrophilic organics consisted of more organic structures which caused high photobacteria acute toxicity, and the structures reacted with chlorine, leading to a decrease in acute toxicity, which compensated the increase in acute toxicity caused by DBPs. In Figure 5, HoA and HoN were observed to exhibit similar photobacteria acute toxicity before and after chlorination, while the formation potential of THMs and HAAs for HoA was about 2 times of that for HoN. The reason might be that HoN also formed other DBPs except THMs and HAAs which were more toxic to photobacteria, thus resulting in comparable acute toxicity with HoA. Wang et al. [10] investigated the genotoxicity of DOM fractions during chlorine disinfection using the $u m u$ test and suggested that the hydrophilic substances were the key fraction involved in decreasing genotoxicity during the chlorination of wastewater with a low $\mathrm{NH}_{3}-\mathrm{N}$ concentration, while the hydrophobic acid fraction of DOM was the key fraction involved in increasing genotoxicity during chlori-
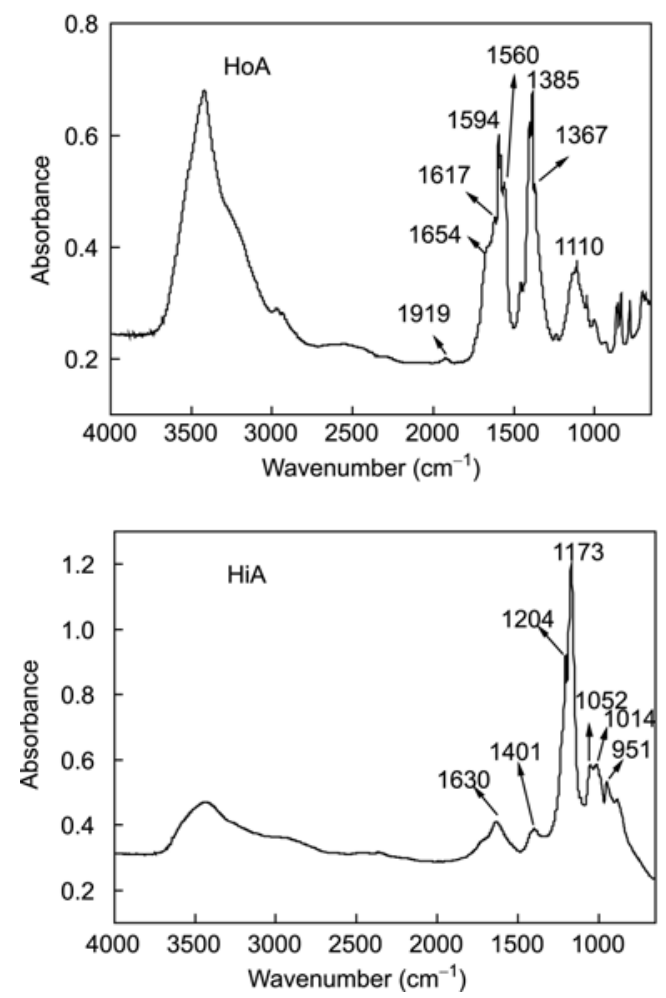

Figure 7 FTIR spectra of DOM fractions. nation of wastewater with a high $\mathrm{NH}_{3}-\mathrm{N}$ concentration.

Ozone pre-oxidation altered the DOM structures and caused a significant increase in the percentage of hydrophilic organics, which remarkably enhanced the photobacteria acute toxicity of DOM.

Therefore, it was essential to compare the characteristics between hydrophobic organics and hydrophilic organics for exploring the organics which caused photobacteria acute toxicity.

\subsection{Characterization of DOM fractions}

The content of $-\mathrm{COOH}$ and ph-OH in DOM fractions was determined by potentiometric titration, as shown in Figure 6.

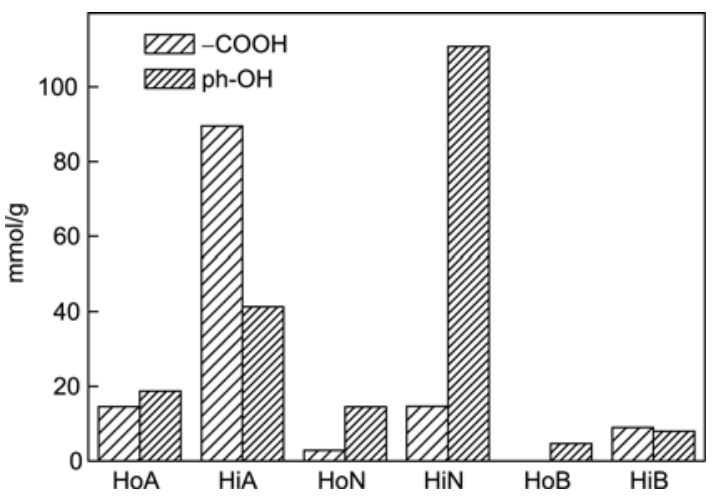

Figure 6 The content of $-\mathrm{COOH}$ and $\mathrm{ph}-\mathrm{OH}$ for DOM fractions.
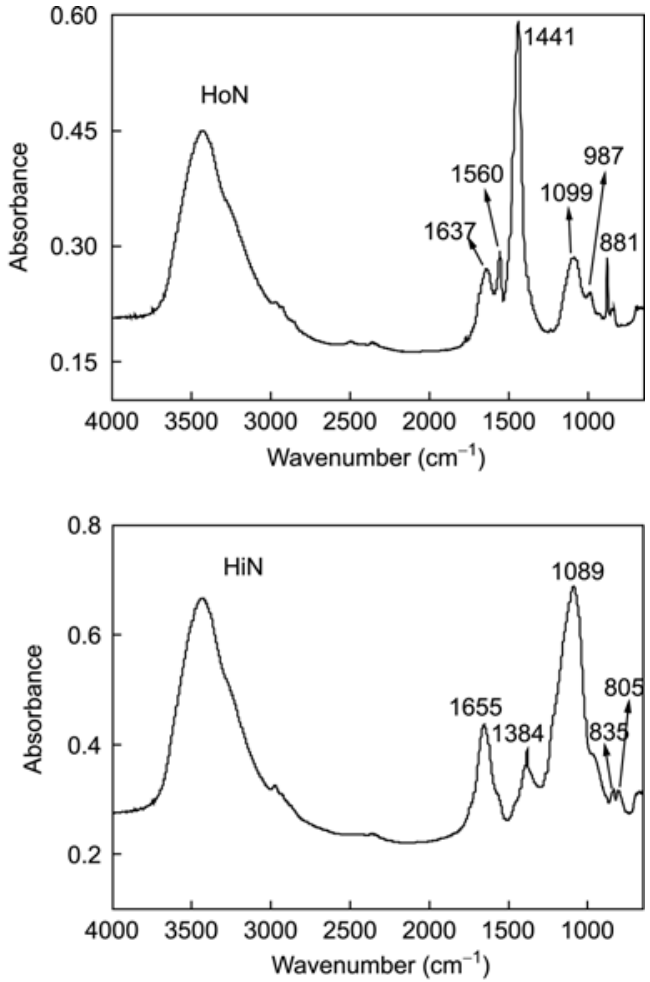
It was observed that the content of - $\mathrm{COOH}$ and ph-OH in hydrophilic fractions was apparently higher than that in the equivalent hydrophobic fractions, i.e. $\mathrm{HiA}>\mathrm{HoA}, \mathrm{HiN}>$ $\mathrm{HoN}, \mathrm{HiB}>\mathrm{HoB}$. Especially for the hydrophilic organics, $\mathrm{HiA}$ showed higher $-\mathrm{COOH}$ content than $\mathrm{HiN}$, whereas $\mathrm{HiN}$ exhibited higher content of ph-OH. Yet, the $\mathrm{HiA}$ and HiN fractions consisted of relatively high content of ph-OH in comparison to hydrophobic organics. It was suggested that the photobacteria acute toxicity was attributed to the ph-OH structure.

In view of the limitation of potentiometric titration analysis, FTIR analysis was used to investigate the structures of DOM (Figure 7). It was observed that there was a similar peak around $1600-1650 \mathrm{~cm}^{-1}$ for the four main fractions of DOM which was defined as the aromatic structures containing $\mathrm{C}=\mathrm{C}$ bonds. Compared to hydrophilic organics, the hydrophobic fractions exhibited a relatively strong peak around $1400-1450 \mathrm{~cm}^{-1}$, indicating aliphatic structures containing $\mathrm{C}-\mathrm{H}$ functional groups, whereas the hydrophilic organics contained relatively strong absorption bands around $900-1200 \mathrm{~cm}^{-1}$ due to hydroxy acids or O-alky group $v(\mathrm{C}-\mathrm{O})$. It was believed that hydrophobic organics contained relatively high content of aliphatic structures, while hydrophilic organics contained relatively high content of aromatic $\mathrm{C}-\mathrm{O}$ groups, such as ph-OH. The difference in organic structures for hydrophobic and hydrophilic organics might cause the variation in photobacteria acute toxicity.

Jennings et al. [11] tested the $\mathrm{EC}_{50}$ of 81 chemicals using Vibrio fischeri. It was observed that aromatic organics showed relatively high toxicity in comparison to aliphatic organics, especially the aromatic organics containing $\mathrm{C}-\mathrm{O}$ groups which exhibited remarkable toxicity. This was consistent with the result in our study.

\section{Conclusions}

Hydrophilic organics in DOM contained relatively high content of ph-OH compared to hydrophobic organics, which caused hydrophilic organics to show significantly high photobacteria acute toxicity. After DOM was ozone preoxidized, the percentage of hydrophilic organics was increased, thus resulting in a significant increase in photobacteria acute toxicity.

This work was supported by the National Natural Science Foundation of China (50538090), Funds for Creative Research Groups of China (50621804), and Scientific Research Program for Saving Water in Beijing: Research and Control on the Safety of Water Reuse.

1 Gagné F, André C, Cejka P, Hausler R, Fournier M, Blaise C. Immunotoxic effects on freshwater mussels of a primary-treated wastewater before and after ozonation: A pilot plant study. Ecotoxicol Environ Saf, 2008, 69(3): 366-373

2 Bellar TA, Lichtenberg JJ, Kroner RC. The occurrence of organohalides in chlorinated drinking water. J Am Water Works Assoc, 1974, 66(12): 703-706

3 Rook JJ. Formation of haloforms during chlorination of natural waters. Wat Treat Exam, 1974, 23(2): 234-243

4 Krasner SW, Weinberg HS, Richardson SD, Pastor SJ, Chinn R, Sclimenti MJ, Onstad GD, Thruston AD. Occurrence of a new generation of disinfection byproducts. Environ Sci Technol, 2006, 40(23): $7175-7185$

5 Zha JM, Wang ZJ. Assessing technological feasibility for wastewater reclamation based on early life stage toxicity of Japanese medaka (Oryzias latipes). Agricult Ecosys Environ, 2005, 107(2-3): 187-198

6 Trevizo C, Nirmalak HN. Prediction of microbial toxicity of industrial organic chemicals. Water Sci Technol, 1999, 39(10-11): 63-69

7 EPA Method 551.1- Determination of chlorination disinfection byproducts, chlorinated solvents, and halogenated pesticides/herbicides in drinking water by liquid-liquid extraction and gas chromatography with electron-capture detection. Environmental Protection Agency. 1995

8 EPA Method 552.3-Determination of haloacetic acids and dalapon in drinking water by liquid-liquid microextraction, derivation, and gas chromatography with electron-capture detection. Environmental Protection Agency. 2003

9 Wang LS, Wei DB, Wei J, Hu HY. Screening and estimating of toxicity formation with photobacterium bioassay during chlorine disinfection of wastewater. J Hazard Mater, 2007, 141(1): 289-294

10 Wang LS, Hu HY, Wang C. Effect of ammonia nitrogen and dissolved organic matter fractions on the genotoxicity of wastewater effluent during chlorine disinfection. Environ Sci Technol, 2007, 41(1): $160-165$

11 Jennings VLK, Rayner-Brandes MH, Bird DJ. Assessing chemical toxicity with the bioluminescent photobacterium (vibrio fischeri): a comparison of three commercial systems. Water Res, 2001, 35(14): 3448-3456 